\title{
The Effect of the Characteristics of Fabrics and Subjective Sensory Images on the Off-line and On-line Preferences of Women's Suit Fabrics
}

This research investigated the influences of structural characteristics such as fabrics, mechanical properties, and subjective sensory images on the off-line and on-line preferences to women's spring/summer suits fabrics to extract the most effective factor towards preference as well as analyze the preferential off-line and on-line differences to predict the exact texture image on-line. Objective evaluations were done for the measurement of the mechanical properties of fabrics using Kawabata's Evaluation System and subjective evaluations were done with 109 female subjects who value the off-line and on-line sensory image of suit fabrics. For statistical analysis, factor analysis, cluster analysis, t-test, ANOVA, and regression were used. The results were as follows. The preference scores on-line were generally higher than those off-line. For the structural characteristics of fabrics, differences of thickness were observed according to preference clusters, and the preference increased as thickness was lowered off-line and on-line. For mechanical properties, WC influenced off-line and on-line preferences. Fabrics with low compression energy were preferred; however, the effect of SMD was observed off-line only. In subjective sensory images, the 'smoothness' image influenced off-line and on-line preferences the most. All sensory images influenced the off-line preferences; however, the effects of 'flexibility' and 'weight' were not shown on-line.

${ }^{*}$ Researcher, Korea Institute of Industrial Technology, Seoul, Korea(nmh@kitech.re.kr)

Associate Professor, Dept. of Fashion Stylist, Hyejeon University, Hongseong, Korea

Key Words: structure of fabric, mechanical property, subjective sensory image, off-line, on-line
Texture image is an important factor for consumer clothing product preferences (especially in women's wear) and is directly related to the purchase of apparel products; subsequently, fabric and clothing manufacturers need to subdivide, specifically analyze, and design the texture image of clothing fabrics. Texture image is strongly influenced by the various physical-chemical characteristics of fabrics such as fiber, yarn, and fabric that influence consumer preferences to apparel products. In addition, there is a need for further information on-line about fabric characteristics of clothing that have texture images differences (compared to off-line) because of the substantial increase in Internet shopping for apparel products recently.

Consumers perceive and select clothing through visual and tactile senses that interpret the texture of fabric. (Han \& Kim, 2006). The texture image consists of sensory and sensibility images. The sensory image is related to the touch sensation of fabrics that can be described by words such as smooth and warm. Sensory image is also very important in consumer preference; subsequently, clothing manufacturers need to analyze and understand consumer preferences in regards to the sensory images of women's wear fabrics and incorporate preferences into the merchandising and marketing of apparel and fabric products.

Many researchers have examined the various aspects of fabric texture images due to the need for 
further information. First, some studies investigated the relationship between texture image and preference for suits fabrics. The study results of relationships between structural characteristics, texture, and preference of fall/winter women's jacket fabrics showed that the weight of women's jacket fabrics indirectly influenced preferences through texture and sensibility; subsequently, the reduction of fabric weight is an important factor in clothing fabric preference (Roh \& Ryu, 2007). In a study on the subjective hand evaluation and preference for men's spring/summer suits fabrics, the 'smoothness' the most influential sensory image on suit fabric preferences (Ryu et al, 2002). Based on men's spring/ summer suit research results, fabrics with higher values for "smoothness" and "flexibility" and lower ones for compression energy and fabric count tended to be preferred (Roh \& Ryu 2005). The sensory image of men's suit fabrics was mostly influenced by smoothness and density and in sensibility images, 'classic' was the most influential factor and 'conservative' influenced negatively on preference (Bae \& Kim, 2003). In addition, the preferred texture image of woolen fabrics was nonrough and soft, uniformly even-surfaced fabrics and 'classic' image influenced the preference and 'elegance' image influenced purchasing intentions (Ko et al., 2003). The analysis of men's spring/summer suits fabrics preferences (according to gender and age) showed that the preference of men in their thirties relates to 'stiffness', 'smoothness', and 'coolness'; however, it relates to 'drapability in the case of men in their twenties (Ju \& Ryu, 2004).

It is important to accurately portray fabric texture on-line, because of the increase in Internet shopping for apparel products. The discord between on-line images and apparel product characteristics is an important problem in Internet shopping (Kim \& Cho, 2004), with fabric texture showing the largest differences (Kim \& Choi, 2002; Cho et al., 2001). It was difficult to estimate the fabric feel from Internet shopping (Lee \& Park, 2004) and most fabrics were evaluated more positively on-line than in person (Kim \& Cho, 2007). Consumer discord can affect the degree of satisfaction after purchase; subsequently, more research about and effective method to transmit the characteristics of very thin, dry, and dense on-line is needed. Further studies will make possible to improve the after purchase satisfaction of consumers and reduce product return rates in ecommerce.

This study investigated the effects of structural and mechanical properties, and subjective sensory images on the preference of women's spring/summer suits fabrics off-line and on-line to extract the most effective factor towards preference; in addition, it analyzed the preferential off-line and on-line differences to predict the texture image on-line exactly. This study will improve customer after purchase satisfaction of apparel products in ecommerce.

\section{METHODS}

Specimens

Among the various women's suit fabrics on the market, the 26 kinds of solid fabrics most preferred in a preliminary survey were used as specimens. Structural characteristics that include thickness, weight, density, weave, and CIE color (JS 555, Colour Techno System Co., Japan) were measured and the fabric specifications are presented in Table 1.

The mechanical properties of the fabrics (that include tensile, bending, shear, surface, compression properties) were measured using Kawabata's Evaluation System (KES)-FB (KATO TECH) under standard conditions.

\section{Evaluation of the Preference of Fabrics}

Subjects The study subjects were 109 female students majoring in clothing and textiles. The evaluation of subjective sensory images were conducted at $\mathrm{C}$ University and $\mathrm{H}$ University in Choong Chung Nam-Do and J University in Gyeonggi-Do, Korea, from May to June, 2010.

Evaluation Procedure The investigation method presents women's suit fabrics off-line and on-line for the evaluation of sensory images and preference. For the off-line evaluation, 26 kinds of fabrics currently on the market were presented for evaluation, using 
Table 1. Fabric Characteristics

\begin{tabular}{|c|c|c|c|c|c|c|c|c|c|}
\hline \multirow{2}{*}{ No. } & \multirow{2}{*}{ Fiber content } & \multirow{2}{*}{$\begin{array}{l}\text { Thickness } \\
\text { (mm) }\end{array}$} & \multirow{2}{*}{$\begin{array}{l}\text { Weight } \\
\left(\mathrm{g} / \mathrm{m}^{2}\right)\end{array}$} & \multirow{2}{*}{$\begin{array}{c}\text { Warp } \\
\text { density (/in) }\end{array}$} & \multirow{2}{*}{$\begin{array}{c}\text { Weft } \\
\text { density (/in) }\end{array}$} & \multirow{2}{*}{ Weave } & \multicolumn{3}{|c|}{ Color } \\
\hline & & & & & & & $\mathrm{L}$ & $\mathrm{a}$ & $\mathrm{b}$ \\
\hline 1 & N55/C40/Pu5 & 0.43 & 27.18 & 34 & 39 & Twill & 68.90 & 0.36 & 5.91 \\
\hline 2 & C60/R40 & 0.28 & 22.45 & 34 & 84 & Twill & 84.24 & 1.81 & 9.50 \\
\hline 3 & P46 R52 Pu2 & 0.33 & 25.49 & 73 & 92 & Satin & 82.20 & -3.27 & 1.96 \\
\hline 4 & Ace62/R38 & 0.37 & 25.97 & 91 & 68 & Satin & 80.33 & 6.37 & 13.99 \\
\hline 5 & $\mathrm{R} 70 / \mathrm{C} 30$ & 0.28 & 21.86 & 80 & 104 & Satin & 71.59 & -0.08 & 5.61 \\
\hline 6 & $\mathrm{P} 34 / \mathrm{C} 62 / \mathrm{Pu} 4$ & 0.33 & 25.96 & 48 & 60 & Satin & 81.20 & 1.43 & 8.96 \\
\hline 7 & R66/C34 & 0.27 & 24.27 & 60 & 75 & Satin & 74.37 & -0.71 & 5.88 \\
\hline 8 & Ace/CTN & 0.29 & 23.83 & 130 & 24 & Satin & 88.95 & -4.06 & 8.65 \\
\hline 9 & $\mathrm{R} 48 / \mathrm{C} 48 / \mathrm{Pu} 4$ & 0.42 & 33.19 & 37 & 78 & Twill & 72.96 & -1.50 & 9.41 \\
\hline 10 & C55/R45 & 0.28 & 26.80 & 35 & 80 & Twill & 80.06 & 0.78 & 10.95 \\
\hline 11 & $\mathrm{R} 70 / \mathrm{F} 17 / \mathrm{C} 13$ & 0.33 & 27.85 & 16 & 58 & Twill & 2.26 & 0.55 & 10.42 \\
\hline 12 & P100 & 0.43 & 25.08 & 22 & 27 & Twill & 12.53 & 2.18 & -4.95 \\
\hline 13 & P65/R35 & 0.36 & 29.54 & 60 & 73 & Satin & 17.01 & 0.64 & -2.31 \\
\hline 14 & P29/R68/Pu3 & 0.39 & 32.27 & 65 & 82 & Satin & 78.97 & -2.26 & 11.64 \\
\hline 15 & $\mathrm{R} 100$ & 0.27 & 25.13 & 64 & 83 & Satin & 41.22 & 2.15 & 3.21 \\
\hline 16 & P100 & 0.37 & 26.59 & 27 & 96 & Twill & 21.54 & 4.48 & -11.92 \\
\hline 17 & R60/P37/Pu3 & 0.38 & 28.41 & 50 & 84 & Twill & 19.05 & 2.61 & -8.24 \\
\hline 18 & C65/L35 & 0.42 & 31.23 & 27 & 47 & Twill & 75.26 & 0.17 & 12.43 \\
\hline 19 & C55/Bem45 & 0.33 & 23.46 & 30 & 67 & Twill & 27.02 & 1.80 & -13.52 \\
\hline 20 & $\mathrm{R} 64 / \mathrm{C} 35$ & 0.25 & 23.74 & 45 & 76 & Satin & 62.92 & 42.27 & 25.10 \\
\hline 21 & N40/R55/Pu5 & 0.53 & 28.89 & 67 & 72 & Satin Var. & 52.75 & -27.50 & 38.64 \\
\hline 22 & $\mathrm{C} 50 / \mathrm{L} 50$ & 0.39 & 31.49 & 18 & 24 & Dobby & 94.05 & -2.96 & 14.00 \\
\hline 23 & Ace67/C30/Pu3 & 0.40 & 36.99 & 65 & 61 & Satin & 73.46 & 0.58 & 16.40 \\
\hline 24 & C55/R42/Pu3 & 0.40 & 26.53 & 25 & 60 & Plain Var. & 102.08 & -4.54 & 7.98 \\
\hline 25 & R43/C49/Pu8 & 0.40 & 23.80 & 88 & 78 & Dobby & 98.61 & -14.78 & 37.29 \\
\hline 26 & $\mathrm{R} 68 / \mathrm{C} 32$ & 0.45 & 27.58 & 70 & 80 & Dobby & 84.40 & -8.36 & 49.59 \\
\hline
\end{tabular}

N: Nylon, C: Cotton, R: Rayon, P: Polyester, Pu: Polyurethane, L: Lamie, Bem: Bembeg Rayon

swatches $20 \mathrm{~cm} \times 30 \mathrm{~cm}$ in size. After the subject observed each fabric using their visual and tactual senses, they were asked to evaluate sensory images and preferences on a 7-point scale (where 1 is Not prefer and 7 is Strongly Prefer). For the on-line evaluation, fabrics were photographed with a digital camera (Nikon COOLPIX P5100, 1.2 mega pixel), and the pictures were presented on a computer monitor (resolution: $1024 \times 768$ pixels) in two different sizes $(5 \times 4 \mathrm{~cm} / 19 \times 14 \mathrm{~cm})$. Pictures were taken under appropriate conditions to represent the distinct characteristics of each fabric. The subjective sensory descriptions consisted of 26 questions and the scale in which the specific value of factors was above 1 was selected. The reliability of each factor verified by Cronbach's alpha.

Statistical Analysis A SPSS Win 13.0 program was used for statistical analysis. They factor analysis was employed to extract subjective sensory image factors and a t-test was used to compare the evaluation scores of off-line and on-line evaluations. A cluster analysis was used to classify fabrics based on preference; subsequently, the off-line and on-line 
sensory image results of the clusters were compared by ANOVA. Regression analysis was used to examine the effects of structural characteristics, mechanical properties, and subjective sensory images on preferences.

\section{RESULTS AND DISCUSSION}

\section{Comparison of the Preference for Off-line and On-line} Evaluations

Comparison of Off-line and On-line Preferences According to Fabric Differences in the results for the preference for the 26 fabrics were compared for the off-line and on-line evaluations and presented in Fig. 1.

The most preferred fabric off-line was rayon/ cotton blended (R 66/C 34) fabric (No. 7), and shiny acetate/rayon blended (Ace 62/R 38) fabric (No. 4) was the most preferred fabric on-line. The preferred on-line and off-line fabrics were No. 3, 4, 7, and 8 (of low thickness), in the whole 26 kinds of fabrics and blended fabric of rayon or acetate fibers. The on-line

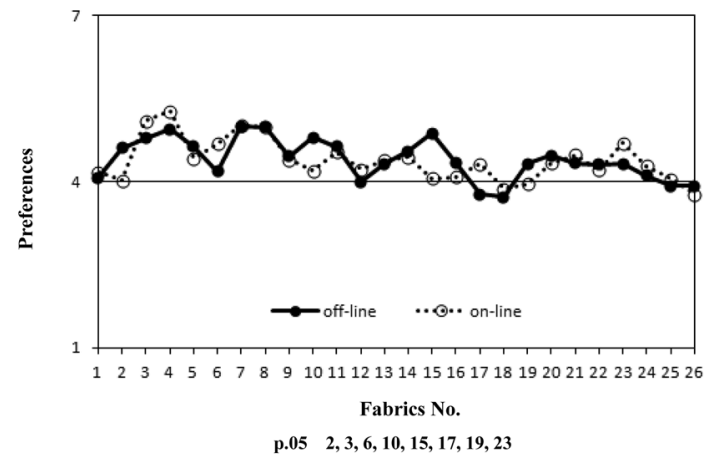

Figure 1. Comparison of Preferences for 26 fAbrics Off-line and On-line and off-line fabrics that showed a low preference score were No. 1, 12, 18, 25 and 26 (of high thickness).

Fabrics that showed the most significant off-line and on-line evaluation differences were No. 2, 3, 6, $10,15,17,19$ and 23 (fabrics blended with $\mathrm{C} / \mathrm{R}, \mathrm{R} / \mathrm{P}$, and Rayon 100\%). The fabric that showed the largest difference between the two kinds of evaluations was No.15 left (Rayon 100\% and low thickness). Therefore, the need to accurately express the texture of thin fabrics on-line has been demonstrated in subsequent studies (Kim \& Cho, 2007).

Preference of Fabrics by Clusters Fabrics were classified using cluster analysis based on a preference score to analyze the differences according to the characteristics of fabrics. Fabrics were grouped into three clusters of high, medium, and low preference.

Table 2 illustrates that off-line fabric Cluster 1 showed a high preference score of 4.89 with a comparatively low thickness, low weight, and mostly blended with rayon was also shown. Off-line fabric Cluster 3 showed a low preference score of 3.93 and was comparatively thick. The on-line high preference cluster had a mean score of 5.09. The fabric characteristics of the high preference cluster were comparatively low thickness of real fabric and mostly blended with rayon or acetate. The low preference cluster had a mean score of 4.05 and the thickness of the real fabrics were comparatively high, but rayon $100 \%$ or polyester $100 \%$ fabrics with low thickness were classified into a low preference cluster on-line.

Comparatively thin and lightweight rayon blended shiny fabrics were preferred for spring/ summer women's suit fabrics; in addition, the online preference scores were generally higher than

Table 2. Comparison of Preference of Fabrics by Clusters

\begin{tabular}{cllcc}
\hline & Preference & \multicolumn{1}{c}{ Fabric Number } & Preference Mean & Preference Range (max.- min.) \\
\hline \multirow{3}{*}{ Off-line } & High & $7,8,4,15,10,3$ & 4.889 & $4.986-4.773$ \\
& Medium & $5,11,2,14,20,9,16,21,13,19,23,22,6$ & 4.417 & $4.626-4.173$ \\
& Low & $24,1,12,25,26,17,18$ & 3.925 & $4.105-3.72$ \\
\hline \multirow{3}{*}{ On-line } & High & $4,3,7,8$ & 5.086 & $5.266-4.973$ \\
& Medium & $23,6,11,21,14,5,9,13,20,17,24$ & 4.447 & $4.689-4.276$ \\
& Low & $12,22,10,1,16,15,25,2,19,18,26$ & 4.047 & $4.213-3.760$
\end{tabular}


Table 3. Structural Characteristics of fabrics by Clusters

\begin{tabular}{|c|c|c|c|c|c|c|c|c|c|}
\hline & \multirow{2}{*}{ Preference } & \multirow{2}{*}{$\begin{array}{l}\text { Thickness } \\
\text { (mm) }\end{array}$} & \multirow{2}{*}{$\begin{array}{l}\text { Weight } \\
\left(\mathrm{g} / \mathrm{m}^{2}\right)\end{array}$} & \multirow{2}{*}{$\begin{array}{c}\text { Warp } \\
\text { Density (/in) }\end{array}$} & \multirow{2}{*}{$\begin{array}{c}\text { Weft } \\
\text { Density (/in) }\end{array}$} & \multirow{2}{*}{ Weave } & \multicolumn{3}{|c|}{ Color } \\
\hline & & & & & & & $\mathrm{L}$ & $\mathrm{a}$ & $\mathrm{b}$ \\
\hline \multirow{4}{*}{ Off-line } & High & 0.458 & 15.988 & 75.500 & 70.333 & 0.833 & 74.521 & 0.210 & 7.440 \\
\hline & Medium & 0.560 & 18.546 & 45.538 & 71.923 & 0.538 & 56.920 & 1.481 & 9.379 \\
\hline & Low & 0.615 & 18.692 & 45.142 & 59.285 & 0.571 & 65.832 & -3.194 & 14.287 \\
\hline & F-value & $11.109^{* * * *}$ & 0.956 & 3.400 & 0.830 & 0.367 & 0.763 & 0.399 & 0.380 \\
\hline \multirow{4}{*}{ On-line } & High & 0.460 & 17.175 & 88.500 & 64.750 & 1.000 & 81.462 & -0.417 & 7.620 \\
\hline & Medium & 0.580 & 20.070 & 50.727 & 73.454 & 0.454 & 57.659 & 1.109 & 11.237 \\
\hline & Low & 0.557 & 16.218 & 40.812 & 64.090 & 0.636 & 62.530 & -1.124 & 10.226 \\
\hline & F-value & $3.961^{*}$ & 2.994 & $6.656^{* *}$ & 0.571 & 0.899 & 0.979 & 0.109 & 0.083 \\
\hline
\end{tabular}

p $0.05,{ }^{* *} \mathrm{p} 0.01,{ }^{* * *} \mathrm{p} 0.001$

Table 4. Mechanical Properties of Fabrics by Cluster

\begin{tabular}{ccccccccccccccccc}
\hline Cluster & LT & WT & RT & EMT & G & 2HG & 2HG5 & B & 2HB & LC & WC & RC & MIU & MMD & SMD \\
\hline High & 0.715 & 12.326 & 50.876 & 7.171 & 0.626 & 0.668 & 1.605 & 0.076 & 0.045 & 0.295 & 0.110 & 40.116 & 0.193 & 0.011 & 2.306 \\
Off-line & Medium & 0.729 & 23.157 & 47.279 & 13.502 & 1.316 & 2.550 & 3.495 & 0.094 & 0.087 & 0.342 & 0.156 & 42.791 & 0.209 & 0.013 & 2.865 \\
Low & 0.738 & 27.917 & 45.714 & 7.171 & 0.622 & 3.838 & 4.314 & 0.118 & 0.112 & 0.377 & 0.162 & 43.551 & 0.202 & 0.017 & 4.881 \\
F-value & 0.319 & 2.319 & 0.796 & 1.982 & 1.868 & 2.351 & 1.790 & 1.383 & 1.505 & 3.226 & $4.455^{*}$ & 0.955 & 0.159 & 1.578 & $9.535^{* * *}$ \\
\hline High & 0.717 & 12.240 & 48.837 & 7.177 & 0.672 & 0.630 & 1.695 & 0.077 & 0.045 & 0.290 & 0.100 & 39.845 & 0.197 & 0.010 & 2.382 \\
On-line & Medium & 0.728 & 27.810 & 45.934 & 16.629 & 1.767 & 4.023 & 4.550 & 0.108 & 0.125 & 0.337 & 0.160 & 42.788 & 0.200 & 0.013 & 2.880 \\
Low & 0.732 & 19.596 & 49.023 & 10.838 & 0.918 & 1.569 & 2.584 & 0.092 & 0.058 & 0.362 & 0.152 & 42.890 & 0.212 & 0.016 & 4.003 \\
& F-value & 0.118 & 2.292 & 0.505 & 2.520 & 3.402 & $3.931^{*}$ & 2.508 & 0.698 & $3.778^{*}$ & 2.155 & $4.347^{*}$ & 0.667 & 0.351 & 2.036 & 2.664 \\
\hline${ }^{*} 0.05,{ }^{* *}$ p0.01, ${ }^{* * *} \mathrm{p} 0.001$ & & & & & & & & & & & & & &
\end{tabular}

those off-line and similar to previous research (Kim \& Cho, 2007). Most fabrics were evaluated more positively on-line than in person; subsequently, consumer discord can affect the degree of after purchase satisfaction.

Comparison of Preferences According to the Structural Characteristics of Fabrics There were differences between clusters according to the characteristics of fabrics; subsequently, the structural fabric characteristics could be analyzed according to clusters using ANOVA. The results are presented in Table 3 and showed significant differences between preference clusters. It was confirmed that the structural characteristics of fabric (that influenced an off-line preference) were thickness. Fabrics with low thickness were grouped into high preference cluster and comparatively thick fabrics were classified into a low preference cluster.

Thickness and warp density influenced on-line preferences. Fabrics with low thickness and high warp density were classified into Cluster 1 and fabrics with high thickness and low warp density were grouped into Cluster 3. Comparatively thin fabrics with high warp density were preferred for spring/summer women's suit fabrics.

Comparison of the Preference according to Mechanical Properties There was a relationship between the characteristics of fabrics and preference scores, the off-line and on-line differences of mechanical properties (according to preference clusters) were analyzed using ANOVA.

By the results of ANOVA in Table 4, there were significant differences in WC (compression energy) and SMD (surface roughness) between the preference 
Table 5. Subjective Sensory Images of Fabrics by Cluster

\begin{tabular}{|c|c|c|c|c|c|}
\hline \multirow{2}{*}{$\begin{array}{l}\text { Sensory } \\
\text { Image }\end{array}$} & \multirow{2}{*}{ Preference } & \multicolumn{2}{|c|}{ Mean } & \multicolumn{2}{|c|}{ F-value } \\
\hline & & Off-line & On-line & Off-line & On-line \\
\hline \multirow{3}{*}{ Stiffness } & High & 3.574 & 3.486 & & \\
\hline & Medium & 3.934 & 3.786 & $14.225^{* * *}$ & $19.299^{* * *}$ \\
\hline & Low & 4.087 & 3.990 & & \\
\hline \multirow{3}{*}{ Flexibility } & High & 4.091 & 4.095 & & \\
\hline & Medium & 3.794 & 3.790 & $5.190^{*}$ & $20.231^{* * * *}$ \\
\hline & Low & 3.700 & 3.624 & & \\
\hline \multirow{3}{*}{ Smoothness } & High & 4.936 & 5.160 & & \\
\hline & Medium & 4.538 & 4.554 & $22.289^{* * * *}$ & $58.115^{* * *}$ \\
\hline & Low & 3.903 & 3.987 & & \\
\hline \multirow{3}{*}{ Warm-Cool } & High & 4.026 & 4.180 & & \\
\hline & Medium & 3.952 & 3.970 & $4.831^{*}$ & $8.716^{* * *}$ \\
\hline & Low & 3.831 & 3.877 & & \\
\hline \multirow{3}{*}{ Elasticity } & High & 3.837 & 4.045 & & \\
\hline & Medium & 3.857 & 3.934 & $3.636^{*}$ & 1.625 \\
\hline & Low & 4.058 & 3.848 & & \\
\hline \multirow{3}{*}{ Weight } & High & 3.652 & 3.328 & & \\
\hline & Medium & 4.085 & 3.887 & 2.334 & $17.138^{\text {*wake }}$ \\
\hline & Low & 4.052 & 3.985 & & \\
\hline
\end{tabular}

clusters off-line and on-line. Fabrics that have a low WC and smooth surface were classified into a high preference cluster and the preference scores were changed according to surface roughness. There were significant differences in preferences according to 2HG (Shear Hysteresis), 2HB (Bending Hysteresis), and compression energy on-line. Fabrics with a low $2 \mathrm{HG}, 2 \mathrm{HB}$, and low compression energy were classified into high preference cluster. Consequently, fabrics that has a low compression energy and smooth surface were preferred for spring/summer women's suit fabrics and the results coincide with preceding research (Ryu \& Roh, 2005), that fabrics with low compression energy were preferred to men's suit fabrics. However, there was a difference between the two kinds of preferences that SMD had no on-line difference; therefore, the surface property was not classified by clusters on-line. It was confirmed that the feeling of surface texture on-line show large differences from real fabrics.
Comparison of the Preference According to Subjective Sensory Image The subjective sensory image scale (in which the specific value of factors is above 1) was selected for the factor analysis; in addition, a Varimax crossing rotation was used. The preference according to the subjective sensory image was compared using ANOVA and the results are presented in Table 5.

The results of ANOVA showed the differences of subjective sensory images between preference clusters off-line and on-line. Subjective sensory images of women's suit fabrics were extracted by factor analysis in a preliminary survey that extracted the six sensory factors of 'stiffness', 'flexibility', 'smoothness', 'elasticity', 'warm-cool', and 'weight'. Preference scores were relative to the scores of 'flexibility', 'smoothness', 'warm-cool', and 'elasticity' images. 'Smoothness' image had the highest preference score and the largest difference between preference clusters off-line and on-line. The results are presented in Fig. 2; in addition, it was found that 


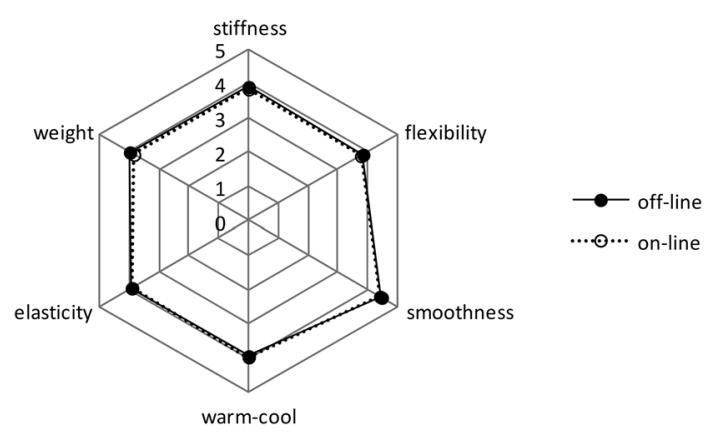

Figure 2. Subjective Sensory Images of Fabrics by Cluster

'smoothness' image showed the highest score in the preference to women's suit fabrics compared with other sensory images of the same cluster.

Preference can be most distinctly classified into clusters by 'smoothness' image. In subsequent research (Bae \& Kim, 2003; Ko et al., 2003; Ryu et al., 2002), 'smoothness' was the important factor for suit fabric preference. In addition, 'elasticity' had no difference between clusters on-line and 'weight' showed a difference on-line only.

\section{The Effects of Objective Properties and Subjective Sensory Images on Preference}

The Effects of Structural Characteristics of Fabrics on Preference The effects of structural characteristics on preference (off-line and on-line) were analyzed by the enter method using multiple regression analysis to investigate the contributiveness of each factor (see Table 6).
By the results of regression analysis, the effects of thickness, weight, and L of color were observed offline and thin and comparatively heavyweight and bright fabrics were preferred. The effect of 'thickness' was especially strong; in addition, the preference increased as thickness decreased. The structural characteristics of fabrics that influenced on-line preferences were thickness, weight, density, and color; subsequently, the fabrics with heavy weight, low thickness, high warp density, and low weft density were preferred. The effect of weight was especially strong. Additionally, the effect of color was also appeared and bright red color was preferred compared with green.

Fabrics with high brightness were preferred for spring/summer women's suit fabrics off-line, and bright green color was preferred on-line compared with red. Then and comparatively heavy and bright fabrics were preferred for spring/summer women's suit fabrics (both off-line and on-line). Additionally, there were differences that the effect of $\mathrm{L}$ of color was shown off-line only; in addition, the effect of color and density were shown on-line.

The Effects of Mechanical Properties of Fabrics on Preference The contributiveness of each factor of mechanical properties to preference (both off-line and on-line) were analyzed using multiple regression analysis (see Table 7).

By the results of regression analysis, the effects of B (bending property), LC, WC, RC (compression

Table 6. Regression Effect of Structural Characteristics on Preferences

\begin{tabular}{|c|c|c|c|c|c|c|c|}
\hline & & \multicolumn{3}{|c|}{ Off-line } & \multicolumn{3}{|c|}{ On-line } \\
\hline & & $B$ & $\beta$ & $t$ & $B$ & $\beta$ & $t$ \\
\hline Thickness & & -4.493 & -0.249 & $-6.508^{* * * *}$ & -2.547 & -0.143 & $-3.742^{* *+* * t}$ \\
\hline Weight & & 0.060 & 0.073 & $2.127^{*}$ & 0.060 & 0.169 & $4.910^{* * * *}$ \\
\hline \multirow{2}{*}{ Density } & Warp & -0.001 & -0.016 & -0.464 & 0.004 & 0.083 & $2.406^{*}$ \\
\hline & Weft & -0.001 & -0.007 & -0.043 & -0.004 & -0.065 & $-2.714^{* *}$ \\
\hline Weave & & 0.085 & 0.043 & 1.289 & 0.080 & 0.041 & 1.231 \\
\hline \multirow{3}{*}{ Color } & $\mathrm{L}$ & 0.003 & 0.051 & $1.893^{*}$ & 0.002 & 0.046 & 1.714 \\
\hline & $\mathrm{a}$ & -0.00 & -0.008 & -0.290 & 0.009 & 0.064 & $2.314^{*}$ \\
\hline & $\mathrm{b}$ & 0.001 & 0.007 & 0.199 & -0.005 & -0.054 & -1.607 \\
\hline
\end{tabular}

"p $0.05,{ }^{* *} \mathrm{p} 0.01,{ }^{* * *} \mathrm{p} 0.001$ 
Table 7. Regression Effect of Mechanical Properties on Preferences

\begin{tabular}{|c|c|c|c|c|c|c|c|}
\hline \multirow{2}{*}{\multicolumn{2}{|c|}{ Mechanical Properties }} & \multicolumn{3}{|c|}{ Off-line } & \multicolumn{3}{|c|}{ On-line } \\
\hline & & B & $\beta$ & $\mathrm{t}$ & B & $\beta$ & $\mathrm{t}$ \\
\hline \multirow{4}{*}{ Tensile } & LT & 1.206 & 0.042 & 0.9437 & 2.612 & 0.093 & $2.050^{*}$ \\
\hline & WT & 0.074 & 0.707 & 1.951 & -0.013 & -0.123 & -0.335 \\
\hline & RT & 0.005 & 0.023 & 0.585 & -0.007 & -0.034 & -0.863 \\
\hline & EM & -0.102 & -0.593 & -1.871 & 0.030 & 0.176 & 0.549 \\
\hline \multirow{3}{*}{ Shear } & G & -0.530 & -0.352 & -1.251 & 0.186 & 0.125 & 0.440 \\
\hline & $2 \mathrm{HG}$ & 0.028 & 0.052 & 0.437 & 0.035 & 0.066 & 0.548 \\
\hline & 2HG5 & 0.005 & 0.009 & 0.047 & -0.099 & -0.185 & -0.945 \\
\hline \multirow{2}{*}{ Bending } & B & 3.206 & 0.102 & $2.403^{*}$ & 0.023 & 0.001 & 0.017 \\
\hline & $2 \mathrm{HB}$ & 1.484 & 0.073 & 0.906 & -1.223 & -0.061 & -0.749 \\
\hline \multirow{3}{*}{ Compression } & LC & -4.277 & -0.183 & $-2.682^{* *}$ & -4.273 & -0.186 & $-2.688^{* *}$ \\
\hline & WC & -3.370 & -0.092 & $-2.724^{* *}$ & -4.170 & -0.115 & $-3.383^{* *}$ \\
\hline & $\mathrm{RC}$ & 0.031 & 0.099 & $2.283^{*}$ & 0.018 & 0.059 & 1.337 \\
\hline \multirow{3}{*}{ Surface } & MIU & -0.043 & -0.001 & -0.028 & 1.657 & 0.045 & 1.075 \\
\hline & MMD & -3.170 & -0.013 & -0.400 & -5.868 & -0.024 & 1.075 \\
\hline & SMD & -0.182 & -0.187 & $-3.750^{* * *}$ & -0.020 & -0.021 & -0.416 \\
\hline
\end{tabular}

p $0.05,{ }^{* *} \mathrm{p} 0.01,{ }^{* * *} \mathrm{p} 0.001$

Table 8. Regression Effect of Subjective Sensory Images on Preferences

\begin{tabular}{lcccccc}
\hline \multirow{2}{*}{$\begin{array}{c}\text { Sensory } \\
\text { Image }\end{array}$} & \multicolumn{3}{c}{ Off-line } & \multicolumn{3}{c}{ On-line } \\
\cline { 2 - 6 } & $\mathrm{B}$ & $\beta$ & $\mathrm{t}$ & $\mathrm{B}$ & \multicolumn{1}{c}{$\beta$} & $\mathrm{t}$ \\
\hline Stiffness & -0.184 & -0.127 & $-5.857^{* * * *}$ & -0.123 & -0.093 & $-4.171^{* * * *}$ \\
Flexibility & 0.154 & 0.125 & $5.604^{* * *}$ & 0.037 & 0.028 & 1.293 \\
Smoothness & 0.481 & 0.398 & $18.613^{* * *}$ & 0.535 & 0.456 & $20.345^{* * *}$ \\
Warm-Cool & 0.128 & 0.089 & $3.896^{* * *}$ & 0.120 & 0.085 & $3.980^{* * * *}$ \\
Elasticity & -0.018 & -0.018 & $-0.867^{* * *}$ & 0.095 & 0.084 & $3.876^{* * *}$ \\
Weight & 0.041 & 0.042 & $1.983^{*}$ & 0.029 & 0.030 & 1.363 \\
\hline
\end{tabular}

$* \mathrm{p} 0.05, * * \mathrm{p} 0.01, * * * \mathrm{p} 0.001$

property), and SMD (surface property) were observed off-line and the effect of surface roughness was strong especially. Thus, the fabrics that have smooth surface, low drapability, and low compression energy (i.e., easy to compress) were preferred. These results coincided with the results of ANOVA of mechanical properties according to preference clusters; in addition, surface roughness shows the largest difference between preference clusters.

The mechanical properties of fabrics that influenced on-line preferences were LT (tensile property), LC, and WC (compression property) (see Table 7). Fabrics that are easy to compress were preferred off-line and on-line; however, the effects of $B$ and SMD were not shown on-line. Supplementary methods are needed to decrease the differences of these properties for off-line and on-line evaluations.

The results coincide with previous studies. The bending property was the most important to subjective sensory images (Kweon et al., 2004); in addition, in the study of Roh and Ryu (2005), fabrics with low compression energy were preferred for 
men's suit fabrics. Surface property appeared as an important preference factor (Ryu et al., 2002; Roh \& Ryu, 2005; Ju \& Ryu, 2004).

The Effects of Subjective Sensory Image of Fabrics on Preference The contributiveness of each subjective sensory image to preference was analyzed using multiple regression analysis off-line and on-line.

In Table 8, all subjective sensory images influenced off-line preferences; in addition, 'smoothness' image influenced preference the most and 'weight' image the least. 'Smoothness' image influenced preference the most on-line as off-line. Subjective sensory images (except 'flexibility' and 'weight') significantly influenced preference that showed the differences with the results off-line. The preference of fabrics changed according to 'smoothness' image. Soft fabrics with smooth surface are appropriate for women's suit fabrics and the results supported those of mechanical properties and previous research (Ko et al., 2003; Roh \& Ryu, 2005; Ryu et al., 2002). The 'flexibility' and 'weight' image of fabric on-line were needed for detailed descriptions about fabric characteristics.

\section{CONCLUSION}

Evaluations of preference for women's suit fabrics were carried out off-line and on-line, and the effects of structural characteristics, mechanical properties of fabrics and subjective sensory images on preference were investigated. The results of this study were as follows.

In the results for the preference for each fabric, the most preferred fabric off-line was rayon /cotton blended; however, shiny acetate/rayon blended fabric was the most preferred fabric on-line. The fabric preferred on-line and off-line had low thickness and blended fabric of rayon or acetate fibers. Fabrics that showed a low preference score on-line and off-line had high thickness.

Fabrics were classified into three clusters according to preference. High preference fabric cluster off-line showed low thickness, low weight, and mostly blended with rayon. The on-line high preference cluster had comparatively low thickness, and mostly blended with rayon or acetate. The preference scores on-line were generally higher than those off-line.

Thickness influenced off-line preferences for the characteristics of fabrics. Fabrics with low thickness were grouped into a high preference cluster and comparatively thick fabrics were classified into low preference cluster. Thickness and warp density influenced on-line preferences.

There were significant differences in mechanical properties between clusters. WC and SMD showed differences between clusters off-line; in addition, fabrics that had a low compression energy and smooth surface were classified into high preference cluster. Fabrics that had a low 2HG, 2HB, and low WC were classified into a high preference cluster online.

The preference scores increased as the scores of 'flexibility', 'smoothness', 'warm-cool' images increased off-line. Preference scores increased as the scores of 'flexibility', 'smoothness', 'warm-cool', 'elasticity' images increased on-line. 'Smoothness' image had the highest preference score and the largest difference between clusters off-line and on-line.

The effects of thickness, weight, and $\mathrm{L}$ of color on preference were observed off-line; in addition, the fabrics of thin and comparatively lightweight as well as bright were preferred. The effect of thickness was especially strong and the preference increased as thickness decreased. The fabrics with comparatively heavy weight, low thickness, high warp density, and low weft density were preferred on-line.

In mechanical properties, the effects of $\mathrm{B}, \mathrm{LC}$, WC, RC, and SMD were observed off-line and the effect of surface roughness was especially strong. Thus, the fabrics that have a smooth surface, low drapability, and low compression energy were preferred off-line. LT, LC, WC were the structural characteristics of fabrics that influenced on-line preferences.

All subjective sensory images influenced off-line preferences; however, 'smoothness' image influenced preference the most and 'weight' image influenced the least. 'Smoothness' image influenced preferences the most on-line as off-line, and it appeared that subjective sensory images (except 'flexibility' and 
'weight') significantly influenced on-line preferences.

There were contrasts between the off-line and on-line results that showed that supplementary methods are needed to decrease the differences and increase the similarities between off-line and on-line evaluations. Consequently, there are many differences between off-line and on-line preferences, and further studies are needed.

First, most fabrics were evaluated more positively on-line than real ones, so discord can affect the degree of satisfaction after purchase. Very thin fabrics showed large differences between two kinds of evaluations; therefore, there is a need to precisely express thickness on-line. Specifically, SMD and surface properties are very important and can affect preference results. A method is needed to accurately express the texture image of fabrics on-line that do not have a distinct surface texture. Next, the 'elasticity', 'flexibility', and 'weight' image of fabric online were needed for detailed descriptions about the characteristics of the fabric. Additionally, preference can be evaluated differently based on L of color offline that points to the need to control brightness to increase similarities in on-line evaluations. These are all areas that warrant further study.

These results of this research equally present useful information to consumers and retailers to improve after purchase product satisfaction and reduce return rates for e-commerce.

\section{REFERENCES}

Bae, H., \& Kim, E. (2003). Texture Image and Preference of Men's Wool/Wool blend Suit Fabrics, Journal of the Korean Society of Clothing and Textiles, 27(1), 1318-1329.

Cho, Y., Lim, S., \& Lee, S. (2001). Apparel purchase behavior among internet shoppers-Focusing on perceived risks-, Journal of the Korean Society of Clothing and Textiles, 25(7), 1247-1257.

Han, E. \& Kim, E. (2006). Classification of Textural Descriptors for Establishing Texture Naming System (TNS) of Fabrics-Textural Descriptions of Women's Suits Fabrics for Fall/Winter Seasons-,
Journal of the Korean Society of Clothing and Textiles, 30 (5), 699-710.

Ju, J., \& Ryu, H. (2004). Preference of S/S men's suit fabrics according to gender and groups, Korean Journal of Human Ecology, 13(4), 609-616.

Kim, C. \& Na, Y. (1999). A Study on Image Scale of the Hand and Sensibility of Silk Woven Fabrics-for Necktie fabric-, Journal of the Korean Society of Clothing and Textiles, 23(6), 898-908.

Kim, S. \& Choi, H. (2002). A Study of the Usage and Sizing Selecting of the Apparels listed in On-line and Catalog Shopping, Journal of the Korean Society of Clothing and Textiles, 26(7), 1015-1025.

Kim, H. \& Cho, S. (2004). The Comparison of Fabric Image between On-line and Off-line by Fabric Types and Characteristics, Korean Journal of Human Ecology, 13(5), 787-798.

Kim, H. \& Cho, S. (2007). The Comparison of Subjective Evaluation of Hand between On-line and Off-line by Structure and Sensibilities of Fabric, Journal of the Korean Society of Clothing and Textiles, 31(1), 1-10.

Ko, S., Yoo, S. \& Kim, E. (2003). DB for the Structural Characteristics, Images and Sensibilities of Fabrics Effects of the Structural Characteristics On the Texture Images of Woolen Fabrics-, Journal of the Korean Society of Clothing and Textiles, 27(5), 533-544.

Kweon, S., Lee, E. \& Choi, J. (2004). A Comparative Study on the Subjective Fabric Hand According to Gender for Winter Sleepwear Fabrics, Fibers and Polymers, 5(1), 6-11.

Lee, K. \& Park, J. (2004). Criteria of Evaluating Clothing and Web Service on Purchase Experience, Journal of the Korean Society of Clothing and Textiles, 28(5), 603-614.

Roh, E. \& Ryu, H. (2005). Visual Texture Image and Preference of Men's suit Fabrics, Korean Journal of the Science of Emotion and Sensibility, 8(2), 117-128.

Roh, E. \& Ryu, H. (2007). Structural Equation Model (SEM) for Constituent Characteristics, Texture, Sensibility and Preference of Fabric (Part 1)-Weight of F/W Women's Jacket Fabrics-, Journal of the Korean Society Clothing and Textiles, 31(8), 1240-1251. 
Ryu, H., Kim, E., Kim, J., Lee, M., Oh, K. \& Yoo, S. (2002). Subjective Hand and Preference of Men's Received April 1, 2012 SpringSummer Suit Fabrics, Proceeding of the 2002 Korean Society of Clothing and Textiles Conference, 561Revised May 9, 2012 561. 